\title{
Understanding granular media: from fundamentals and simulations to industrial application
}

\author{
André Katterfeld $^{1}$. Christopher Wensrich ${ }^{2}$
}

Published online: 31 October 2017

(c) Springer-Verlag GmbH Germany 2017

\begin{abstract}
Academic and industrial research on the behaviour of granular material is increasingly supported by numerical simulations. Such simulation environments are not only a vital tool in design and analysis, but also a key link between physicists and engineers. This Topical Collection includes a range of papers that use simulation to study granular matter from the micro- and macroscopic behaviour of particles to the behaviour of large industrial or geomechanical systems. These contributions enhance our understanding of basic physical effects and processes such as heat transfer, agglomeration and screening but also give essential parameters for the simulation of industrial systems, or, at least, guide the strategies required to provide realistic simulation results. The collection focuses on the presentation of (a) new coupled simulation methods to consider the interaction of granular bodies with structural or fluid systems (b) new findings for the consideration of particle shape and particle size distributions within simulations, (c) acoustic wake agglomeration, and (d) gravitational flow in geomechanics.
\end{abstract}

Keywords Granular matter - Discrete Element Method . DEM - Coupling · DEM calibration · FEM · DNS · CFD · Agglomeration

This article is part of the Topical Collection on: Understanding granular media - from fundamentals and simulations to industrial application.

Christopher Wensrich

christopher.wensrich@newcastle.edu.au

1 Institut für Logistik und Materialflusstechnik, Otto von Guericke Universität Magdeburg, Universitätsplatz 2, 39106 Magdeburg, Germany

2 School of Engineering, University of Newcastle, University Dr, Callaghan, NSW 2308, Australia

\section{Introduction}

Granular media provide for an interesting and wide research area due to the complex physical mechanisms that can occur within the smallest sample of the most common good. A teaspoon of sugar contains hundreds of thousands of highly irregularly-shaped particles that undergo complex interactions with their neighbours. In many ways, a high-fidelity understanding of even this 'simple' system is still beyond our abilities.

Outside of this academic interest, granular media have a high economic importance in society in fields such as energy generation, construction, food production and in the general process industries. This broad interest creates an environment of interaction between engineers, physicists and mathematicians, all focused on the common goal of understanding these materials. Simulation now forms the key meeting-point between these different disciplines.

Perhaps the most important simulation technique developed for the analysis of particulate systems is the Discrete Element Method (DEM). Since its inception in the late 1970s [1], this technique has grown to be central to the field with a continued exponential growth in application amongst a long-term increasing interest in granular materials in general [2]. This growth of DEM was first highlighted by Zhu et al. [3] and it has continued over the last decade. To illustrate, Fig. 1 shows the number of papers per year found by Google Scholar for the search terms "Discrete Element Method" and "DEM" since 1979. Over this period, the rate of publication of papers involving DEM has roughly doubled every 3.3 years. The seminal work in this area by Cundall and Strack [1] now has in excess of 11,000 citations (Google Scholar).

This situation has been aided by the advent of accessible tools for numerical simulation and 'cheap' computational 


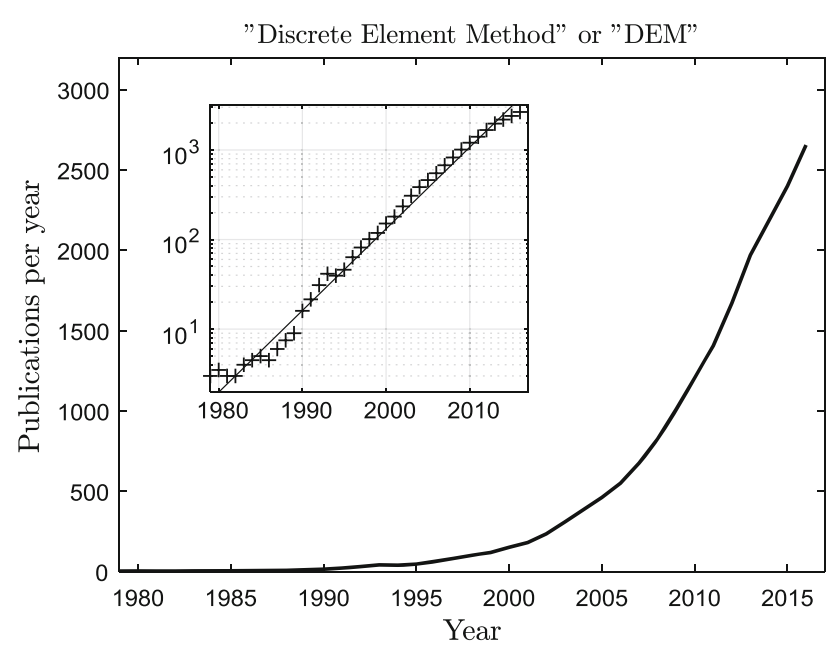

Fig. 1 The rate of publication of scientific papers involving DEM since 1979 (Google Scholar). The rate of increase is approximately an order of magnitude per decade

power that have brought DEM to the attention of practicing engineers. As a common language between physicists and engineers, this now makes the results of fundamental research work (e.g. the development of new contact models) directly applicable to industry related questions. These problems typically involve large systems with complex boundary conditions stemming from detailed wall geometries. Such complex boundary conditions cannot easily be considered in detail by theoretical approaches based on continuum mechanics.

As the level of idealisation shrinks with increasing computational power, more and more complex problem can be simulated. DEM simulations now often involve hundreds of thousands of particles (or more) and the coupling of several simulation methods to examine more than one phase of media. (e.g. the movement of particles within gas or fluid flows, or the interaction with structures). In this environment, the right modelling approach and the appropriate selection of simulation parameters has become increasingly important.

It is interesting to notice that this general trend can be found throughout the many areas of application of DEM. Traditionally, research fields like geomechanics, process engineering or conveying technologies have their own specific problems, however the use of the same numerical methods and the questions related to these tools now intimately link these fields together.

Such a trend can be also found in the work presented at preeminent scientific conferences in the area, where DEM features heavily. In 2015 such a meeting took place when the 8th International Conference of Conveying and Handling of Particulate Solids (CHoPS) was hosted in Tel Aviv, Israel. This conference series now draws in participants from across various areas in engineering, the engineering sciences and physics to discuss the common language of numerical simulation of granular systems and its application to industry problems. The Topical Collection within this Special Issue drew inspiration from this event, and it later formed the initial point in the collection of individual contributions. These contributions contain novel research results, and were peer reviewed as regular high-quality research papers.

The papers in this collection present new approaches, new coupling methods and new ways for the parametrisation of DEM models from a range of research fields and applications. The scale of interest varies from the localised behaviour of micrometer particles to geological features. Some papers are focused on increasing our fundamental understanding of physical processes from an academic point of view, while others detail methods for determining simulation parameters or findings that guide the modelling of industrial systems. The common ground in each case is the use of numerical simulation as a tool to capture the physics of these systems.

Several important trends are noted. These include coupling of DEM to other methods, consideration of particle size and shape distributions, calibration of DEM parameters, and universal analysis methods.

\section{Coupling of DEM simulations}

Increasing computational power allows the coupling of different simulation methods to consider complex interactions between different systems (or components within a single system), particularly the response of granular systems to fluid flows. In many systems this can be the dominant influence on particle behaviour.

The idea of considering the interaction of fluid and particle phases by coupling different models together is not new; various approaches to the problem have been reviewed by Zhou et al. [3,4]. From this point of view, the approach is now coming-of-age with attention now turning to its application to understanding physical and industrial processes. This Topical Collection contains two such papers that use coupled CFD-DEM simulations for the investigation of heat transfer in granular systems [5] and a DNS-DEM simulation to study the manipulation of particle flows through inertial focusing in microchannels [6].

The Topical Collection also contains a paper focussing on the coupling of FEM-DEM models to consider the deformation of structures due to the interaction between particles and mechanical handling systems [7]. This work highlights the important fact that DEM is increasingly used for the determination of design loads in complex industrial systems. Only the development of two-way couplings such as this allows for a direct consideration of the influence of each component 
of the system on the other (i.e. the response of the flow to structural deformation and vice versa).

\section{Consideration of particle size and shape distributions}

The generation of a defined particle size distribution is a key features of most DEM codes. Although implicitly trusted by most researchers, the inbuilt particle generation algorithms can have issues with specific arrangements like binary mixtures with large size ratios. Chang and Deng [8] examine these issues in their contribution focused on developing a particle generation algorithm for sand-silt mixtures.

The assumption of spherical particles is still prevalent in the area. Spheres have many advantages in terms of fast and efficient detection and handling of contacts, however there are often situations where caution is required. Various alternatives now exist for modelling shape. Examples include;

- The multisphere/clustering/clump approach where defined shapes are constructed from conglomerations of several overlapping spheres [9],

- Rounded polyhedral particles [10], and,

- Particle shapes defined by generating functions such as superquadrics and spheroids [11].

Many of these alternatives have existed for some time, but their usage is limited due to the additional computational power required. For this reason, it is still quite common to use rolling friction as a proxy for shape [12-14]. In some practical situations there is no alternative, and the effects of particle shape must be approached and treated pragmatically.

Sieving processes, such as that examined by Chen et al. [15], provide such an example. In this contribution, the influence of different approaches to modelling shape are examined in terms of the results of sieving simulations. The implication of this work in terms of industrial application is obvious; realistic simulations rely on getting many details correct, but the importance of a robust calibration process remains paramount.

\section{Calibration of DEM parameters}

Continuing on the theme of calibration, all practical DEM simulations of real particle systems involve some degree of idealisation. Real particle systems involve trillions (or more) particles with shapes we can only hope to model accurately. From this perspective, contact model parameters should be viewed as caricatures of reality with the actual focus on maintaining a correspondence between the bulk behaviour in simulation and the real system. In the end, this can only be assured through a vigorous calibration process involving experimental investigation. This vital role of calibration as a part of the model setup/preparation can be observed as a running theme throughout the Topical Collection (e.g. $[7,9,15])$.

A wide variety of calibration tests are now described in the literature [16], however the determination of contact parameters from bulk behaviour is far from trivial. Optimisation algorithms are now becoming increasingly popular for this task $[17,18]$. From this perspective, Elskamp et al. [9] presents a calibration procedure for spherical and nonspherical particles based on Genetic Algorithms.

\section{Universal analysis method}

The breadth in the areas of application of DEM is on full display within this Topical Collection. This ranges from investigations involving process technologies at the microscale (e.g. [5,19]) to tools aimed at guiding the design of large scale materials handling equipment [7] and applications in geosciences [20].

All papers in this collection serve to demonstrate that DEM has become a modern, reliable analysis tool of broad utility in both engineering and science.

\section{Compliance with ethical standards}

Conflict of interest The authors declare that they have no conflict of interests.

\section{References}

1. Cundall, P.A., Strack, O.D.: A discrete numerical model for granular assemblies. Geotechnique 29(1), 47-65 (1979)

2. Luding, S.: Die Physik kohäsionsloser granularer Medien. Logos Verlag, Berlin (1998)

3. Zhu, H.P., Zhou, Z.Y., Yang, R.Y., Yu, A.B.: Discrete particle simulation of particulate systems: theoretical developments. Chem. Eng. Sci. 62(13), 3378-3396 (2007)

4. Zhu, H.P., Zhou, Z.Y., Yang, R.Y., Yu, A.B.: Discrete particle simulation of particulate systems: a review of major applications and findings. Chem. Eng. Sci. 63(23), 5728-5770 (2008)

5. Oschmann, T., Kruggel-Emden, H.: Numerical and experimental investigation of the heat transfer of spherical particles in a packed bed with an implicit 3D finite difference approach. Granul. Matter 19(3), 47 (2017)

6. Udono, H., Sakai, M.: A numerical study on dynamic inertial focusing of microparticles in a confined flow. Granul. Matter 19(4), 79 (2017)

7. Dratt, M., Katterfeld, A.: Coupling of FEM and DEM simulations to consider dynamic deformations under particle load. Granul. Matter 19(3), 49 (2017)

8. Chang, C., Deng, Y.: A particle packing model for sand-silt mixtures with the effect of dual-skeleton. Granul. Matter 19(4), 80 (2017)

9. Elskamp, F., Kruggel-Emden, H., Hennig, M., Teipel, U.: A strategy to determine DEM parameters for spherical and non-spherical particles. Granul. Matter 19(3), 46 (2017) 
10. Eliáš, J.: Simulation of railway ballast using crushable polyhedral particles. Powder Technol. 264(6), 458-465 (2014)

11. Podlozhnyuk, A., Pirker, S., Kloss, C.: Efficient implementation of superquadric particles in Discrete Element Method within an opensource framework. Comput. Part. Mech. 4(1), 101-118 (2017)

12. Ai, J., Chen, J.F., Rotter, J.M., Ooi, J.: Assessment of rolling resistance models in discrete element simulations. Powder Technol. 206(3), 269-282 (2011)

13. Wensrich, C.M., Katterfeld, A.: Rolling friction as a technique for modelling particle shape in DEM. Powder Technol. 217, 409-417 (2012)

14. Wensrich, C.M., Katterfeld, A., Sugo, D.: Characterisation of the effects of particle shape using a normalised contact eccentricity. Granul. Matter 16(3), 327-337 (2014)

15. Chen, W., Donohue, T., Katterfeld, A., Williams, K.: Comparative discrete element modelling of a vibratory sieving process with spherical and rounded polyhedron particles. Granul. Matter 19(4), 81 (2017)
16. Coetzee, C.J.: Review: calibration of the discrete element method. Powder Technol. 310, 104-142 (2017)

17. Rackl, M., Hanley, K.J.: A methodical calibration procedure for discrete element models. Powder Technol. 307, 73-83 (2017)

18. Benvenuti, L., Kloss, C., Pirker, S.: Identification of DEM simulation parameters by artificial neural networks and bulk experiments. Powder Technol. 291, 456-465 (2016)

19. Kačianauskas, R., Maknickas, A., Vainorius, D.: DEM analysis of acoustic wake agglomeration for mono-sized microparticles in the presence of gravitational effects. Granul. Matter 19(3), 48 (2017)

20. Xu, S., Suorineni, F.T., An, L., Li, Y.: A study of gravity flow principles of sublevel caving method in dipping narrow veins. Granul. Matter 19(4), 82 (2017) 